\title{
Chapter 6 \\ Applying the Dual-Spacecraft Approach to the Swarm Constellation for Deriving Radial Current Density
}

\author{
Hermann Lühr, Patricia Ritter, Guram Kervalishvili and Jan Rauberg
}

\begin{abstract}
One of the Swarm prime mission goals is the estimation of ionospheric currents. Of particular interest in this context are field-aligned currents (FACs). In order to improve our ability of determining FACs, two of the Swarm spacecraft are orbiting side-by-side separated only by $1.4^{\circ}$ in longitude. This close-formation flight enables the application of Ampère's integral law to magnetic field measurements for estimating radial currents. From experience gained in space we can state that most reliable results are obtained in the auroral region. Here the spacing of the measurement quad and the size of current structures match best. In the vicinity of the poles, close to the orbital crossovers, spacecraft separations become too small for reliable gradient measurements. At low latitudes the separation becomes largest $(\sim 150 \mathrm{~km})$. Here certain FAC features, e.g. associated with plasma instabilities and disturbances exist, which cannot be analysed reliably with the dual-SC approach. However, mid-latitude large-scale currents like the inter-hemispheric FACs can be recorded reliably by the Swarm mission. Besides presenting some measurement examples special emphasis is put on the discussion of underlying assumptions and on the limitations of the approach.
\end{abstract}

\subsection{Introduction}

Measurements of current distributions in space are normally based on the interpretation of magnetic fields they generate. In general, the magnetic field caused by an electric current can be predicted everywhere. But reversely, from single-satellite magnetic field measurements the current distribution cannot be deduced uniquely. For obtaining still useful results, for example the current geometry can serve as $a$ priori information. Such assumptions are commonly based on physical considerations. In case magnetic field measurements from more points are available, well distributed in space, fewer assumptions are required. This argument has been an

H. Lühr $(\bowtie) \cdot$ P. Ritter · G. Kervalishvili · J. Rauberg

GFZ, German Research Centre for Geosciences, Potsdam, Germany

e-mail: hluehr@gfz-potsdam.de 
important motivation for deploying multiple-spacecraft constellations (e.g. 4 Cluster, 3 Space Technology 5 (ST-5), 3 Swarm, 4 Magnetospheric Multi-Scale (MMS)) flying in close formation.

For a local determination of the full current density vector simultaneous magnetic field measurements from at least four well-spaced points are needed. Realising this approach was one of the prime goals of the Cluster mission. The technique for estimating currents from the four Cluster spacecraft was developed and described before the mission (e.g. Dunlop et al. 1988), and later a number of studies made use of the so-called curlometer technique (e.g. Dunlop et al. 2002; Vallat et al. 2005). The same approach is now applied to MMS data (e.g. Zhao et al. 2016). Experience has shown that this 4-point method also has its limits (e.g. Dunlop et al. 2016). In particular it depends on a suitable spacing between the spacecraft. Most favourable is a regular tetrahedron. But the spacecraft formation evolves over an orbit, thus can only be optimal within a certain region. An important assumption of the method is that the magnetic field changes only linearly between the measurement points. A significant contribution to field curvature can come from the Earth's main field. Subtracting a suitable main field model from the magnetic field readings before interpretation will reduce that problem. Furthermore, it is requires that the inter-spacecraft separations have to be significantly smaller than the dimensions of the dominant current system. An attempt in that direction is realised by the MMS mission with spacecraft distances of only some tens of kilometres (e.g. Eastwood et al. 2016). However, in such cases demands are high on accurate positioning of the spacecraft and on the precision of the measurements. Estimates of typical FAC spatial scales have been derived from the three ST-5 spacecraft taking measurements at varying separations (e.g. Slavin et al. 2008).

When coordinated magnetic field measurements from less than four satellites are available assumptions on the current characteristics have to be made (e.g. Vogt et al. 2013). In the topside ionosphere, where the Swarm satellites orbit, the highly anisotropic conductivity distribution is an important constrain. The field-aligned conductivity is several orders of magnitudes higher than the transverse one. This implies that any current detected at that altitude is presumably field-aligned. Another assumption concerns the stationarity of current structures, including both their motion and temporal evolution. This requirement has to be related again to the orbital dynamics of the Swarm spacecraft. Any motion of a current sheet is typically much slower than the satellite velocity of $7.6 \mathrm{~km} / \mathrm{s}$. Similar care has to be taken when combining measurements in a solution from slightly different times. Larger-scale (>150 km) field-aligned current (FAC) structures, however, have been found to be stable typically for longer than one minute (e.g. Gjerloev et al. 2011; Lühr et al. 2015a).

Taking these characteristics into account, reliable FAC density estimates can be obtained in the ionosphere, especially at high latitudes, by a pair of spacecraft flying side-by-side like the Swarm A and C constellation. In the section to follow, we first provide a general introduction into the estimation of electric current density from magnetic field measurements. A detailed description of FAC determination from the lower pair of Swarm satellites is given in Sect. 6.3. For a selected number of cases we present examples of FAC observations in Sect. 6.4. The subsequent section 
provides a detailed discussion of uncertainties inherent to the actually implemented dual-SC method for FAC estimates. Finally we summarise the main points and draw conclusions.

\subsection{Current Estimates from Satellites}

The typical approach for deriving current estimates from magnetic field measurements is to make use of Ampère's law, the curl-B relation

$$
\operatorname{curl} \mathbf{B}=\mu_{0} \mathbf{j}
$$

where $\mathbf{j}$ is the current density, $\mu_{0}$ is the permeability of free space and $\mathbf{B}$ the magnetic field generated by the current. For the vertical current component, $j_{Z}$, Eq. (6.1) reduces to

$$
j_{Z}=\frac{1}{\mu_{0}}\left(\frac{\partial B y}{\partial x}-\frac{\partial B x}{\partial y}\right)
$$

where $B x$ and $B y$ are the horizontal magnetic field components in northward and eastward directions of a local Cartesian coordinate system, respectively. At auroral latitudes, beyond $60^{\circ}$ magnetic latitude (MLat), the field lines are almost vertical exhibiting inclination angles larger than $75^{\circ}$. Therefore $j_{Z}$ represents quite closely the field-aligned current (FAC) density. FACs can be derived more precisely when Mean-Field-Aligned (MFA) coordinates are used in Eq. (6.2). In the MFA frame the $\mathrm{z}$ component is aligned with the ambient magnetic field, the y component, pointing eastward, lies within the horizontal plane and is perpendicular to the mean field, and the $\mathrm{x}$ component, pointing outward/upward, completes the right-handed triad.

\subsubsection{Single-Satellite Field-Aligned Current Estimate}

In case magnetic field recordings are available only from a single satellite, data sampled along the track have to be used for estimating $j_{Z}$. This requires further simplifications of Eq. (6.2) (e.g. Lühr et al. 1996).

$$
j_{Z}=\frac{1}{\mu_{0}} \frac{\Delta B y^{\prime}}{v \Delta t}
$$

Here $B y^{\prime}$ is the horizontal component perpendicular to the FAC sheet normal, $v$ is the orbital velocity component aligned with the sheet normal and $\Delta t$ is the time difference between adjacent measurements. Spatial field gradients required for Eq. (6.2) are derived from subsequent readings of $B y^{\prime}$ assuming stationarity of the currents. 
Furthermore, FACs are believed to be organised in elongated sheets. The orientation of the current sheet has to be determined by other techniques (e.g. minimum variance analysis, Sonnerup and Cahill 1967). Often it is simply assumed that the satellite crosses the current sheet at a right angle. Any deviation from this geometric assumption causes an underestimation of current density.

In summary, the reliability of FAC density estimates from single-satellite data depends on a number of important assumptions:

1. Stationarity of current density during the crossing

2. Organisation of FACs in elongated sheets

3. Knowledge of the current sheet orientation.

\subsubsection{Multi-satellite Current Estimates}

Significantly improved current density estimates can be achieved when simultaneous magnetic field measurements at well-spaced distances are available. In the case of multiple data points Ampère's law in integral form is preferably used

$$
j=\frac{1}{\mu_{0} A} \oint \vec{B} \cdot d \vec{\ell}
$$

where $d l$ is the path element along the closed contour, $A$ is the encircled area, and $j$ is the mean value of the current density component normal to the plane. Simultaneous measurements from at least three points are needed to calculate a ring integral. For this special case Eq. (6.4) can be written in discrete form

$$
j=\frac{1}{2 \mu_{0} A}\left[\left(\vec{B}_{1}+\vec{B}_{2}\right) \cdot \vec{l}\left(Q_{1}, Q_{2}\right)+\left(\vec{B}_{2}+\vec{B}_{3}\right) \cdot \vec{l}\left(Q_{2}, Q_{3}\right)+\left(\vec{B}_{3}+\vec{B}_{1}\right) \cdot \vec{l}\left(Q_{3}, Q_{1}\right)\right]
$$

where $\boldsymbol{B}_{n}$ are the magnetic field vectors at the corners and $l(Q n, Q m)$ are the path elements connecting the points, see Fig. 6.1. The area $A$ can be calculated as

$$
A=\frac{1}{2}\left|\vec{l}_{a} \times \vec{l}_{b}\right|
$$

where $l_{a}$ and $l_{b}$ are any two legs $l(Q n, Q m)$ of the triangle. The obtained $j$ represents the mean current density component normal to the encircled plane, and it is assigned in time and space to the barycentre of the triangle.

In cases where simultaneous measurements at four points in space are available (like Cluster or MMS) three such triangles can be formed. In principle this allows to derive current density components in three independent directions and thus to build the complete current density vector. The feasibility of this current estimation 
Fig. 6.1 Magnetic field measurements at the three corners of the triangle are used for estimating the mean current density flowing through encircled area

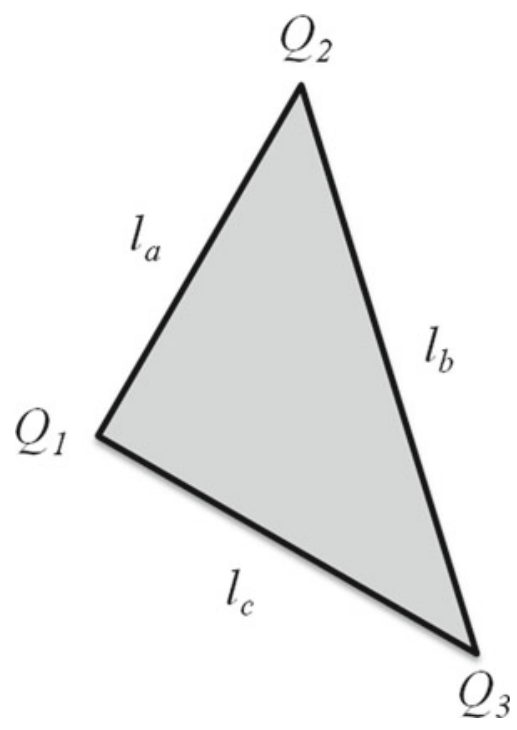

technique was demonstrated successfully in several cases (e.g. Dunlop and Balogh 2005; Zhao et al. 2016). Further details of the so-called 'curlometer technique' can be found e.g. in Dunlop et al. (2002) and references therein.

Occasionally the three Swarm spacecraft pass the auroral region in a close formation. For some of these incidences the 3-point current analysis could be applied, see Dunlop et al. (2015) and Chapter 5. This analysis has also been expanded by including time-shifted measurement points for building up a multi-point virtual constellation, allowing to derive a complete set of current components (Dunlop et al. 2015). Unfortunately, only very few cases are so far available for such an analysis. The orbital plane of Swarm B drifted quickly away from that of the Swarm A \& C pair.

However, significant improvements over single-satellite current estimates can already be achieved when magnetic field measurements from a pair of satellites, flying side-by-side, are considered. In general, such a formation is suitable for deriving radial currents. In the topside ionosphere, where Swarm satellites are orbiting, the direction of currents is closely controlled by the orientation of the geomagnetic field because of the high electric conductivity along field lines. For that reason the dual-satellite configuration is also well suited for estimating FAC density at high and middle latitudes.

The idea of deriving radial current density estimates from the lower pair of Swarm spacecraft has been intensively tested in preparation for the mission as part of an ESAsponsored study. With the help of a closed-loop model simulation the reliability of the dual-spacecraft (dual-SC) radial current approach could be demonstrated. The field-aligned currents predicted by a magnetohydrodynamic (MHD) model were compared with the current estimates derived from related magnetic fields sampled along virtual orbits of Swarm. The improvement in reproducing the input FACs by 
the dual-SC technique as compares to the single-satellite approach was confirmed by this study. More details about this modelling study can be found in the Final Report (Vennerstrøm et al. 2005) or in Ritter and Lühr (2006).

Based on these promising results it was decided by ESA to compute the radial current density routinely from the magnetic field measurements of the two Swarm spacecraft flying side-by-side and to offer the results as a standard Swarm Level 2 product.

\subsection{The Swarm Dual-SC Current Estimate Approach}

The three Swarm spacecraft (Friis-Christensen et al. 2006) where launched on 22 November 2013 by a single rocket into a high-inclination $\left(\sim 87.5^{\circ}\right)$ orbit. After the commissioning phase was completed an orbit manoeuvre campaign followed for setting up the final constellation. Since 17 April 2014 Swarm A and C are flying side-by-side separated by about $1.4^{\circ}$ in longitude at the equator at an initial altitude of about $460 \mathrm{~km}$. Swarm B is cruising somewhat higher at about $510 \mathrm{~km}$ and a larger inclination $\left(\sim 88^{\circ}\right)$. For that reason the orbital plane of Swarm B precesses slightly slower than that of the lower spacecraft pair, gradually building up a local time difference between the orbits.

With Swarm A and C, cruising at the same altitude, there exists the risk of collision at the orbital crossover points near the poles. Therefore the spacecraft are slightly phased along the orbit. Their equator crossing times differ within the range 5-10 s. This time difference has to be taken into account when interpreting the zonal gradient.

Our aim is to derive the radial current density by integrating the magnetic field around a rather regular quad, as depicted in Fig. 6.2. The area does not need to be strictly rectangular, but a large area-to-circumference ration is weighting down the influence of data noise and biases. As a first step, our approach determines the exact times of passing the orbital crossovers of the two spacecraft at high latitudes. From the time difference the orbital phasing $(\sim 7 \mathrm{~s})$ is derived. Swarm A is used as reference satellite, and the readings of Swarm $C$ are shifted in time by the phasing. In this way a synchronous side-by-side configuration is achieved. The synchronisation is updated on every passage of the North or South Pole and kept constant over half an orbit. The along-track field gradients, from Point 1 to Point 2 or from Point 4 to Point 3, are obtained by considering measurements $5 \mathrm{~s}$ later. This time step is equivalent to a distance of $38 \mathrm{~km}(7.6 \mathrm{~km} / \mathrm{s}$ times $5 \mathrm{~s})$. In cross-track direction the distance, equivalent to $1.4^{\circ}$ in longitude, varies from $155 \mathrm{~km}$ at the equator to zero at the crossover points. An approximately quadratic shape of the quad is achieved around latitudes of $75^{\circ}$, which occurs within the auroral region. Here we can obtain the most accurate results. The position and time stamp of the derived mean radial current density is assigned to the barycentre of the quad (see Fig. 6.2). Here again Swarm A acts as reference.

The basis for the current density estimates are the observed magnetic field deflections, which are caused by the currents. For obtaining them we have to remove the 
Fig. 6.2 Sketch of the quad of four measurement points used in the Swarm mission for calculating radial currents. The resulting mean current density is assigned to the barycentre (cross)

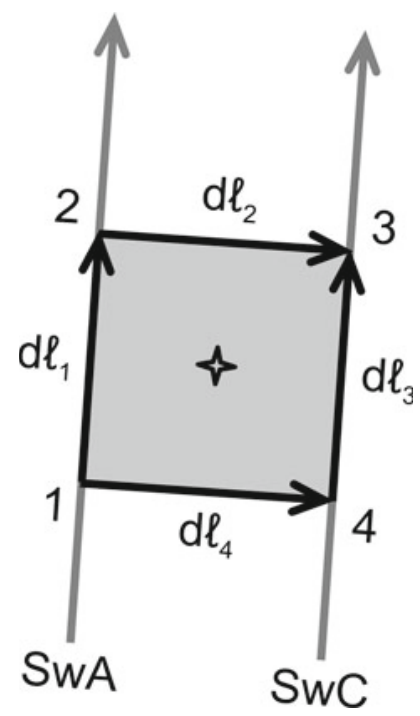

contributions of the core field, the crustal magnetisation and large-scale magnetic current systems from the original magnetic field readings:

$$
B^{R E S}=B^{O B S}-B^{C O R}-B^{L I T}-B^{E X T}
$$

where $B^{O B S}$ represent the original readings, $B^{C O R}$ is the core field, $B^{L I T}$ is the lithospheric field and $B^{E X T}$ is caused by magnetospheric currents. All these three contributions are represented nowadays reliably by magnetic field models like CHAOS-6 (e.g. Finlay et al. (2016) and Chapter 12). In all subsequent analyses $B^{R E S}$ is used. For the sake of clarity we will omit the superfix (RES) in the following.

The radial current density is calculated with the help of Ampére's integral law, as shown in Eq. (6.4). Here we apply a discrete form for solving the ring integral

$$
j_{r}=\frac{-1}{2 \mu_{0} A}\left[\left(\vec{B}_{A T 1}+\vec{B}_{A T 2}\right) \cdot d \vec{l}_{1}+\left(\vec{B}_{C T 2}+\vec{B}_{C T 3}\right) \cdot d \vec{l}_{2}-\left(\vec{B}_{A T 3}+\vec{B}_{A T 4}\right) \cdot d \vec{l}_{3}-\left(\vec{B}_{C T 4}+\vec{B}_{C T 1}\right) \cdot d \vec{l}_{4}\right]
$$

where $B_{A T n}$ and $B_{C T n}$ are the along-track and cross-track magnetic field components aligned with the respective connections between the quad points, $d l_{n}$ are the corresponding path elements. The calculation of the area $A$ will be introduced further down.

The magnetic field observations are given in the North-East-Center (NEC) frame. However, for solving Eq. (6.7) we need the field components aligned with the connecting lines between corner points. This requires defined rotations of the horizontal components. 


$$
B_{A T n}=B_{N E C x} \cos \alpha_{n}+B_{N E C y} \sin \alpha_{n} \quad B_{C T n}=B_{N E C x} \cos \beta_{n}+B_{N E C y} \sin \beta_{n}
$$

The rotation angles $\alpha_{n}$ and $\beta_{n}$ can be deduced from the position data of the satellites. Here we have to take into account that the spacecraft locations are generally given in Earth-fixed coordinates, but ionospheric currents are better organised in the local time (LT) frame. In order to account for this effect, we introduce an LT-related longitude, $\lambda$

$$
\lambda=\varphi+(\mathrm{t} / 86400) * 360^{\circ}
$$

where $\varphi$ is the geographic longitude and $t$ is the time in seconds of a day.

Figure 6.3 shows how the angles $\alpha_{n}$ and $\beta_{n}$ between the path elements and the direction to the pole are defined. The calculation of these angles is based on spherical geometry. The basic equation is the sine formula for triangles on a sphere:

Fig. 6.3 Definition of angles $\alpha$ and $\beta$ between the four route elements and the connections to the geographic pole (modified after Ritter et al. 2013)

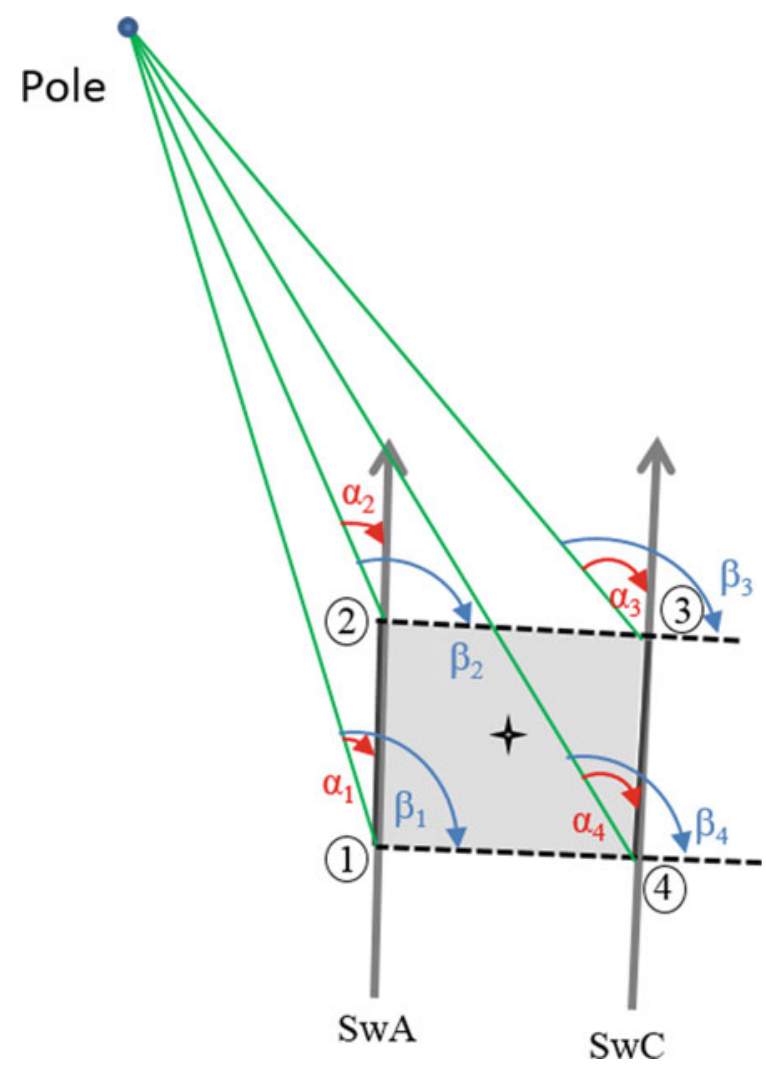




$$
\frac{\sin (\operatorname{arc} \gamma)}{\sin \gamma}=\frac{\sin (\operatorname{arc} \delta)}{\sin \delta}
$$

where $\operatorname{arc} \gamma$ and arc $\delta$ represent the sides of the triangle opposite to the respective angles $\gamma$ and $\delta$. For computing arc $\gamma$ and arc $\delta$ we make use of the great circle distances. The angles $\alpha_{n}$ and $\beta_{n}$ are then computed as:

$$
\begin{aligned}
& \alpha_{1}=\arcsin \left[\frac{\sin \left|\lambda_{2}-\lambda_{1}\right| \cdot \sin \theta_{2}}{\sin \left[\arccos \left(\cos \theta_{1} \cos \theta_{2}+\sin \theta_{1} \sin \theta_{2} \cos \left(\lambda_{2}-\lambda_{1}\right)\right)\right]}\right] \\
& \alpha_{2}=\arcsin \left[\frac{\sin \theta_{1}}{\sin \theta_{2}} \sin \alpha_{1}\right] \\
& \alpha_{4}=\arcsin \left[\frac{\sin \left|\lambda_{3}-\lambda_{4}\right| \cdot \sin \theta_{3}}{\sin \left[\arccos \left(\cos \theta_{3} \cos \theta_{4}+\sin \theta_{3} \sin \theta_{4} \cos \left(\lambda_{3}-\lambda_{4}\right)\right)\right]}\right] \\
& \beta_{1}=\arcsin \left[\frac{\alpha_{3}=\arcsin \left[\frac{\sin \theta_{4}}{\sin \theta_{3}} \sin \alpha_{4}\right]}{\sin \left[\arccos \left(\cos \theta_{1} \cos \theta_{4}+\sin \theta_{1} \sin \theta_{4} \cos \left(\lambda_{4}-\lambda_{1}\right)\right)\right]}\right] \\
& \beta_{4}=\arcsin \left[\frac{\sin \theta_{1}}{\sin \theta_{4}} \sin \beta_{1}\right] \\
& \beta_{2}=\arcsin \left[\frac{\sin \left|\lambda_{3}-\lambda_{2}\right| \cdot \sin \theta_{3}}{\sin \left[\arccos \left(\cos \theta_{3} \cos \theta_{2}+\sin \theta_{3} \sin \theta_{2} \cos \left(\lambda_{2}-\lambda_{3}\right)\right)\right]}\right] \\
& \beta_{3}=\arcsin \left[\frac{\sin \theta_{2}}{\sin \theta_{3}} \sin \beta_{2}\right]
\end{aligned}
$$

In all equations $\theta_{n}$ represent the co-latitude at the quad corner points. The angles $\alpha_{n}$ are close to zero on the orbital upleg arc and close to $180^{\circ}$ on the downleg arc, whereas $\beta_{n}$ stay close to $90^{\circ}$ on both sides of the orbit. At polar regions the angles vary strongly. As an example, the evolution of the angles $\alpha_{1}$ and $\beta_{1}$ over an orbit are presented in Fig. 6.4 (lower panel). The angles at the other corner points exhibit quite similar values.

As a final step for completing the radial current calculation according to Eq. (6.7) the integration area needs to be computed. To be more general we allow for a certain distortion of the area framed by the path elements.

$$
A=\frac{1}{2}\left[\left(d l_{2}+d l_{4}\right)\left(d l_{1} \sin \left(\frac{1}{2}\left(\beta_{1}-\alpha_{1}+\beta_{2}-\alpha_{2}\right)\right)+d l_{3} \sin \left(\frac{1}{2}\left(\beta_{3}-\alpha_{3}+\beta_{4}-\alpha_{4}\right)\right)\right)\right]
$$

When approaching the poles the cross-track distances, $d l_{2}$ and $d l_{4}$, become progressively smaller, see Fig. 6.4 (top panel). As a consequence, the vanishing area, $A$, 

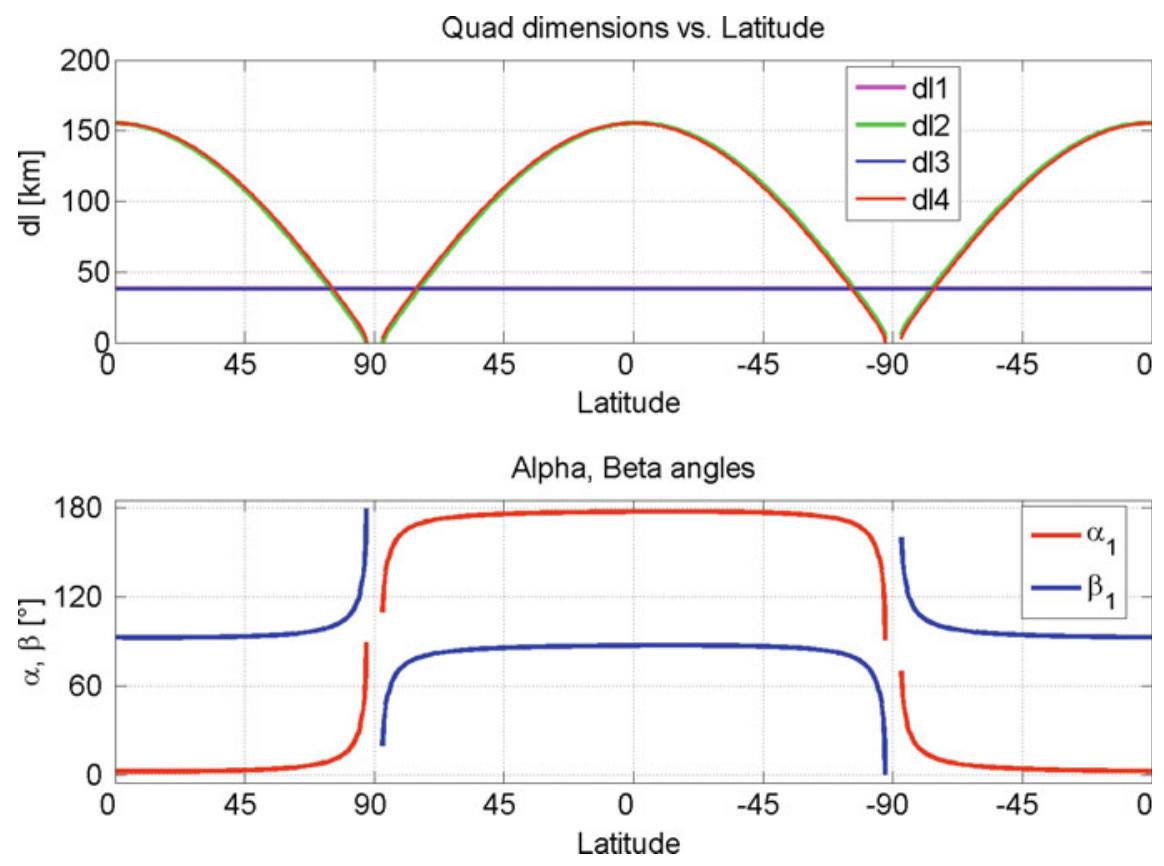

Fig. 6.4 Top: Variation of route elements over an orbit. The along-track elements, $d l 1, d l 3$ stay constant whereas the cross-track ones, $d l 2, d l 4$ become progressively smaller towards the poles. Due to an orbital inclination of less than $90^{\circ}$, the close vicinity of the poles is not sampled. Bottom: Variation of the angles $\alpha$ and $\beta$ along the orbit. Here $\alpha 1$ and $\beta 1$ have been chosen as examples for the four angles (modified after Ritter et al. 2013)

make the current estimates unreliable. For that reason no radial currents are calculated from Swarm data at latitudes beyond $86^{\circ}$.

Since the current estimates are based on observations at only four discrete points, the method needs to consider aliasing effects. According to the sample theorem the signal should not contain spatial variations with wavelength shorter than twice the distance between any two corner points. In an attempt to satisfy this requirement we have low-pass filtered the magnetic field residuals with a $-3 \mathrm{~dB}$ cutoff frequency of $50 \mathrm{mHz}$. This corresponds to an along-track wavelength of about $150 \mathrm{~km}$. Our assumption is that spatial structures have comparable wavelength in along-track and cross-track directions. With this approach the spatial aliasing effect should be avoided, at least at auroral latitudes.

In particular at auroral latitudes field-aligned currents are of great interest because they transfer energy and momentum from the magnetosphere into the ionospherethermosphere system. We derive FAC density estimates by mapping the radial current density, $j_{r}$, onto the field direction. This is done by considering the magnetic field inclination, $I$ : 


$$
j_{\|}=\frac{-j_{r}}{\sin I}
$$

In order to avoid unrealistic FAC values near the equator, where the inclination angle approaches zero, no FAC values are calculated for $|I|<30^{\circ}$. This corresponds to a range of $\pm 15^{\circ}$ in magnetic latitude MLat. Vertical currents, however, are available through all latitudes. Please note that a positive sign represents downward FACs in the north and upward FACs in the southern hemisphere.

A detailed description of the processing algorithms used for the actual Swarm Level 2 product "FACxTMS_2F" can be found in Ritter et al. (2013).

\subsection{Examples of Swarm FAC Estimates}

ESA's standard Swarm Level 2 data processing provides current densities derived by the dual-SC approach from Swarm A and C both in radial direction and field-aligned at $1 \mathrm{~s}$ cadence. However, due to the low-pass filtering of magnetic field data with a cut-off period of $20 \mathrm{~s}$ only large-scale radial current structures are recovered with horizontal wavelengths larger than $150 \mathrm{~km}$ when considering the spacecraft velocity of $7.5 \mathrm{~km} / \mathrm{s}$.

For completeness, single satellite FAC and radial current densities from all three spacecraft are also calculated and made available. In this case the $1 \mathrm{~Hz}$ data are not filtered reflecting all the spatial scales of FAC structures. However, it has to be kept in mind that the reliability of small-scale FAC estimates is questionable because the temporal variability of small-scale FACs may be quite significant and violate one of the basic assumptions in Sect. 6.2.1, as was shown by Lühr et al. (2015a).

Subsequently we will present a few examples of FAC estimates and compare single and dual-SC results. In order to make the curves comparable, the single-satellite results shown here were low-pass filtered in the same way as the dual-SC ones. Generally we find good agreement between the individual FAC estimates from the two spacecraft, Swarm A and C, and also with the dual-SC results. In particular, this is true for the auroral oval region in the northern hemisphere. Figure 6.5 shows an example from a polar passage, crossing first the night-time auroral oval before flying into the pre-noon sector. The current sheet signatures observed are typical for these areas. The large amplitudes at higher latitudes can be related to Region 1 (R1) FACs. Please note that positive values represent downward FACs in the northern hemisphere. At certain locations the dual-SC results reveal larger peak values than the single-satellite estimates. This underestimation of the current density is probably due to a non-perpendicular orientation of the current sheet with respect to the satellite track.

In spite of the generally good agreement there are a number of cases where significant differences appear between FAC estimates from single satellite and dualSC approach. Two such examples are shown in Fig. 6.6. At auroral latitudes, a good agreement between the two techniques is observed also here, but at higher latitudes, 

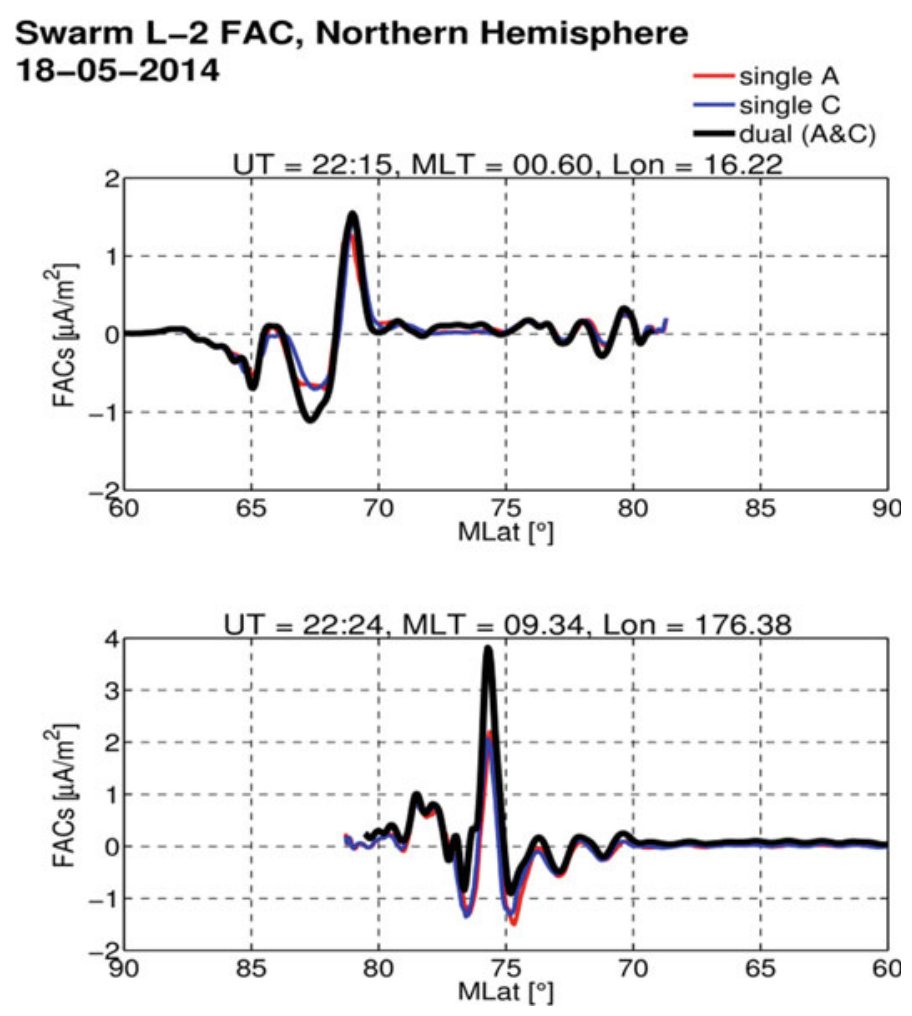

Fig. 6.5 Examples of field-aligned current measurements at auroral latitudes. Individual FAC estimates from the satellites Swarm A and Swarm C are compared with the dual-SC results. In the northern hemisphere positive values represent downward FACs

beyond $75^{\circ}$ MLat, clear differences are obvious. Lühr et al. (2016) investigated the properties of such events showing discrepancies in the polar cap region. In some of these cases simultaneous observations by DMSP satellites are available, providing also auroral images. One example from the southern hemisphere is shown in Fig. 6.7. In the top left frame, the differing FAC estimates are plotted. Discrepancies appear poleward of $70^{\circ}$ MLat. Below is the auroral image shown, taken by DMSP F16 and mapped onto magnetic coordinates. To this image we added the Swarm orbit track. The satellite pair moves from the early morning sector towards noon. Due to the large offset between geographic and geomagnetic poles in the southern hemisphere, Swarm skims the oval beyond $75^{\circ}$ MLat at a small angle. This prevents the single satellite technique to obtain reliable values for FAC densities. The particle instruments on DMSP (see Fig. 6.7, right frames) support the existence of the FAC sheets derived with the dual-SC approach. They show enhanced electron precipitation collocated with the currents. In particular during times of northward interplanetary magnetic field (IMF) orientation, FACs are known to appear frequently in the polar cap (see 

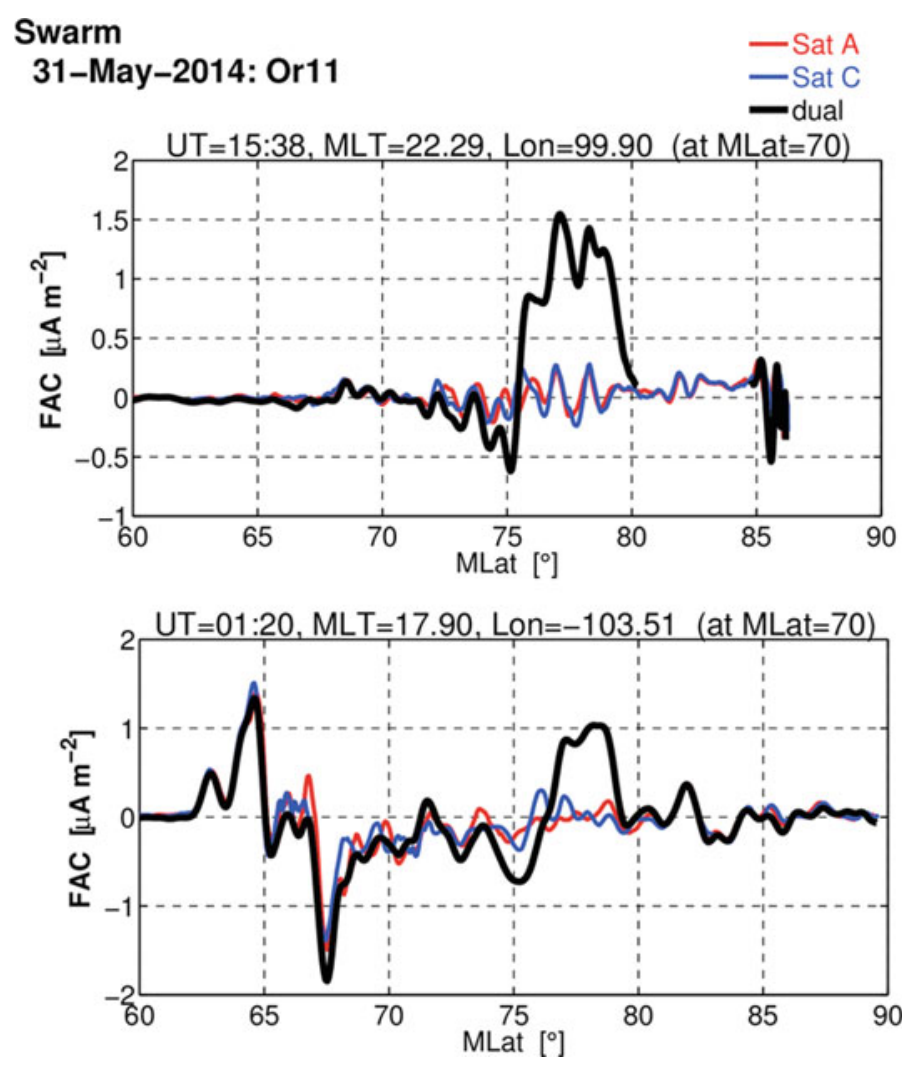

Fig. 6.6 Examples of observations where significant differences are found between FAC estimates from single satellites and the dual-SC approach. At auroral latitudes the agreement is good. Deficits of the single-satellite results appear generally in the polar cap region

Lühr et al. 2016). For those conditions, the dual-SC technique promises to provide new information.

\subsection{Assessing the Uncertainties of FAC Estimates}

The Swarm dual-SC approach for estimating the radial current density can be regarded as a major progress compared to the traditional way of determining FAC density from single-satellite magnetic field measurements. Still, the dual-SC approach is not perfect, and we will discuss here some of the limitations.

Ambient magnetic field: Before starting with the uncertainties, we'd like to highlight one big advantage of the dual-satellite approach, which overcomes a serious drawback of the single satellite approach. The influence of the various sources of the 


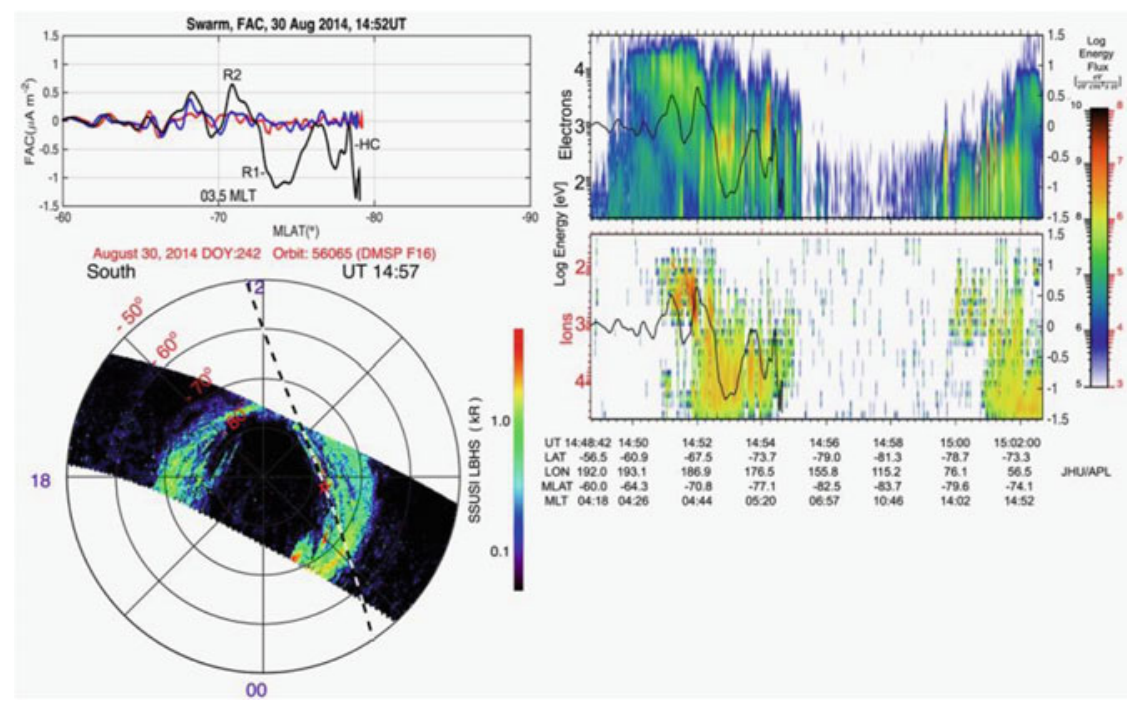

Fig. 6.7 Composite plot of Swarm and DMSP observations of a polar cap event in the Southern Hemisphere. Field-aligned currents along the Swarm track are presented in the top left graph. The black curve shows dual-SC FAC and the red and blue curves present single satellite results of Swarm A and C results, respectively. Auroral images and the Swarm orbit track are illustrated below. The diagrams on the right side show precipitating particle spectrograms with FAC profiles over-plotted (black curve), electrons in the top panel and ions at the bottom (modified after Lühr et al. 2016)

ambient magnetic fields is often ignored when considering error sources. Different from single satellite results, the radial current density derived from a ring integral is hardly influenced by magnetic fields of distant sources: for a field configuration that can be described by a scalar potential, the sum of observations along a closed loop approaches zero. This means that any deficits in main magnetic field removal or in correcting the ring current effect should not compromise the dual-SC current estimates. This holds also for the magnetic effect of the auroral electrojet. Contributions of the electrojets to the magnetic field readings of low-Earth orbiting satellites, such as Swarm, are often underestimated. They can affect significantly the determination of the FAC sheet orientation, for example when employing a minimum variance analysis. In the case of a single-satellite current determination the crossing angle has to be known for a correct estimate. Different from that the skewing angle of the FAC sheet with respect to the flight direction is of no concern for the dual-SC approach. This feature is particularly useful for studies of the southern auroral region, as has been shown above.

Linearity of the magnetic field: One important assumption for the dual-satellite approach is that the magnetic field between the corner points varies linearly. Only in that case the mean value of the two readings at adjacent points represents the average over the entire distance. It is known that the magnetic field at auroral latitudes can vary significantly on scales much shorter than the typical size of our integration 
quad (about $50 \mathrm{~km}$ ). For mitigating this problem, short-wavelength magnetic field structures are filtered out before processing. We apply a low-pass filter with a $-3 \mathrm{~dB}$ cutoff period of $20 \mathrm{~s}$. This period corresponds to a wavelength of $150 \mathrm{~km}$ in alongtrack direction. If Swarm crosses a pair of anti-parallel FAC sheets, the upward and downward FACs detected are separated at least by $75 \mathrm{~km}$. Spatial gradients related with structures of this size can be considered as sufficiently linear over distances smaller than $50 \mathrm{~km}$.

Temporal stability of the current sheets: There is also a certain requirement on the temporal stability of the current structures because measurements separated by $5 \mathrm{~s}$ are combined in a single solution. In the auroral region frequently intense kinetic Alfvèn waves can be observed. They typically have periods of $10 \mathrm{~s}$. With our $20 \mathrm{~s}$ low-pass filter we efficiently suppress these waves. For larger FAC structures Lühr et al. (2015a) have shown that they are stationary for $1 \mathrm{~min}$ or longer. Here again the applied filter is considered to be sufficient for solving the stability problem. Any linear change in FAC intensity is averaged and attributed to the centre time between the two measurement points $5 \mathrm{~s}$ apart. Also Le et al. (2009) studied temporal variability based on ST-5 data for meso-scale $(\sim$ a few $100 \mathrm{~km})$ and large scale $(\sim 1000 \mathrm{~km})$ FACs. They confirm a typical stability of about $1 \mathrm{~min}$ for FAC of these scales.

Stationarity of the current sheets: The situation is slightly different when the current sheet is moving. In that case the amplitude of current density will not change much, but the current sheet appears broader when the satellites move in the same direction as the sheet and narrower when both move in opposite directions (Doppler Effect). As a consequence, the total current is overestimated or underestimated by the ratio $\frac{v_{\text {sat }}+v_{\text {sheet }}}{v_{\text {sat }}}$ or $\frac{v_{\text {sat }}-v_{\text {sheet }}}{v_{\text {sat }}}$ for parallel or opposite velocities, respectively. A typical sheet velocity may amount to a few hundred meters per second. This has to be compared to $7.6 \mathrm{~km} / \mathrm{s}$ of the satellite speed in orbit. Therefore resulting uncertainties are generally below $10 \%$. Since independent magnetic field measurements from two spacecraft (Swarm A and C) are available, it is in principle possible to check for the motion of the current sheet. A suitable strategy is to determine first the orientation of the current sheet at the two spacecraft and then check the differences in crossing time. Either they are consistent with passages through a static tilted sheet or a motion along the sheet normal has to be considered to make the times fit. Wang et al. (2009) performed a detailed study on FAC motion at auroral latitudes based on ST-5 measurements. They confirmed that most determined FAC speeds are found in the range $50-200 \mathrm{~m} / \mathrm{s}$. Typically the speed increases with higher magnetic activity.

Orientation of the current sheet: In Sect. 6.4 we had claimed that the orientation of the current sheet does not influence the resulting current density, as long as the horizontal scales of the sheet are larger than the dimensions of the measurement quad. In order to demonstrate that, two explicit examples are presented here. For the sake of traceability we make some simplifying assumptions, which however, do not limit the generality of the results.

The current is flowing in an infinitely long vertical sheet of width $2 w$. We assume homogenous current distribution within the sheet. Therefore only a transverse magnetic field is induced that varies linearly from $B_{T}$ to $-B_{T}$ through the sheet, as shown 


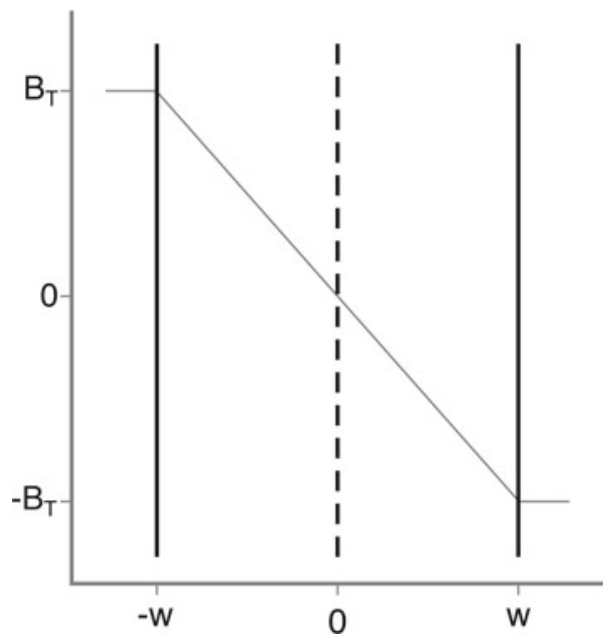

Fig. 6.8 Magnetic field variation across a homogeneous FAC sheet of $2 \mathrm{w}$ width. The tangential field component, $\mathrm{B}_{\mathrm{T}}$, varies linearly from one side of the sheet to the other

in Fig. 6.8. In the first example the measurement quad is located well within the current sheet that is oriented at an arbitrary angle $\varphi$ with respect to the geometry of the quad. Figure 6.9 (top) presents for this case the measurement configuration. Important quantities are the distances $u_{n}(n=1,2,3,4)$ from the measurement points to the outer border of the current sheet. With some simple geometric analysis we obtain:

$$
\begin{aligned}
& u_{1}=\left(d l_{1}+d\right) \sin \varphi \\
& u_{2}=d \sin \varphi \\
& u_{3}=d \sin \varphi+d l_{2} \cos \varphi \\
& u_{4}=\left(d l_{1}+d\right) \sin \varphi+d l_{2} \cos \varphi
\end{aligned}
$$

where $d$ is the along-track distance from Point 2 to the border of the current sheet. Here it is assumed that $d l_{1}=d l_{3}, d l_{2}=d l_{4}$ and the area $A=d l_{1} . d l_{2}$. Both conditions are well approximated by the implemented measurement strategy.

The current density is calculated according to Eq. (6.7). For that purpose the magnetic field components $B_{A T}$ and $B_{C T}$ at the corner points, aligned with the path elements, are needed. They can generally be written as:

$$
B_{A T n}=B_{T n} \cos \varphi \quad B_{C T n}=B_{T n} \sin \varphi
$$

where $B_{T n}=B_{T}\left(1-u_{n} / w\right)$. When inserting the field components into Eq. (6.7) the current density $j$ results 

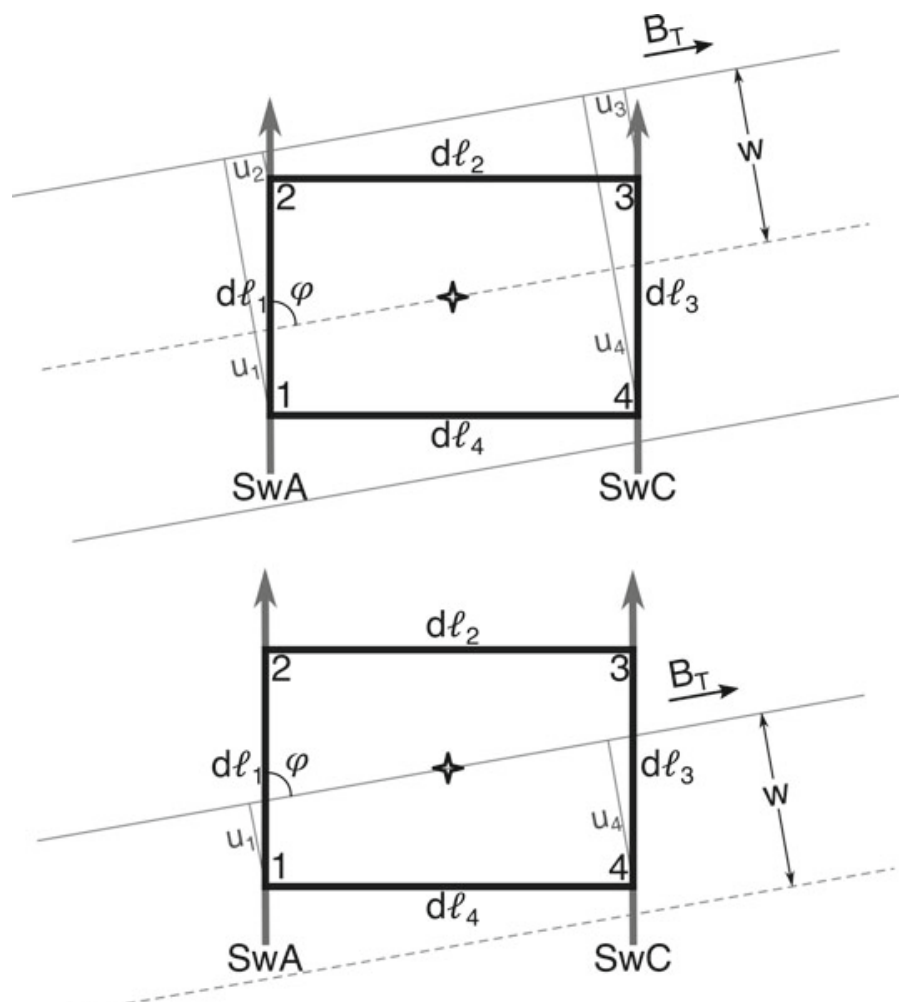

Fig. 6.9 Schematic drawings of two FAC measurement configurations. In the top part the measurement quad is located fully within the current sheet. In the bottom part only half of the quad is engulfed by the FAC sheet

$$
\begin{gathered}
j=\frac{B_{T}}{2 w \mu_{0} A}\left[\left(d l_{2} \cos \varphi+d l_{2} \cos \varphi\right) d l_{1} \cos \varphi+\left(d l_{1} \sin \varphi+d l_{1} \sin \varphi\right) d l_{2} \sin \varphi\right] \\
j=\frac{B_{T}}{2 w \mu_{0} A}\left[2 d l_{1} d l_{2}\right]
\end{gathered}
$$

According to our definition, the term in brackets represents just two times the area A. We thus finally get:

$$
j=\frac{B_{T}}{w \mu_{0}}
$$

which is the actual current density within the FAC sheet. The result is independent of the orientation angle, $\varphi$, and of the distance, $d$, to the border. 
The second example considers a situation where the measurement quad has been shifted partly out of the current sheet, as shown in Fig. 6.9 (bottom). For convenience, the border runs through the center of the quad, but tilted at an arbitrary angle $\varphi$. Now only the distances $u_{l}$ and $u_{4}$ are of concern

$$
\begin{aligned}
& u_{1}=\frac{d l_{1}}{2} \sin \varphi-\frac{d l_{2}}{2} \cos \varphi \\
& u_{4}=\frac{d l_{1}}{2} \sin \varphi+\frac{d l_{2}}{2} \cos \varphi
\end{aligned}
$$

Correspondingly, the magnetic field components at the corners amount to:

$$
B_{A T 1,4}=B_{T}\left(1-u_{1,4} / w\right) \cos \varphi \quad B_{C T 1,4}=B_{T}\left(1-u_{1,4} / w\right) \sin \varphi
$$

While for the other two corners outside the current sheet we get:

$$
B_{A T 2,3}=B_{T} \cos \varphi \quad B_{C T 2,3}=B_{T} \sin \varphi
$$

When inserting the field components from (6.25) and (6.26) into Eq. (6.7) we obtain for the current density:

$$
j=\frac{B_{T}}{2 w \mu_{0} A}\left[d l_{2} \cos \varphi d l_{1} \cos \varphi+d l_{1} \sin \varphi d l_{2} \sin \varphi\right]
$$

resulting in

$$
j=\frac{B_{T}}{2 w \mu_{0}}
$$

which is just half the value of (6.24). This makes sense since only half of the quad area is engulfed by the current sheet. Also in this case, no dependence on the tilt angle, $\varphi$, results. The independence of our dual-SC current determination technique on the orientation of the sheet can also be shown for more complicated current density profiles. But for them a numerical modelling is recommended, which goes beyond the scope of this chapter.

Measurement accuracies: One important assumption for the dual-SC approach is the accuracy of magnetic field measurements at the two spacecraft. The differences in field readings at two positions are interpreted in terms of currents. Any bias or uncertainty in the scaling factor will cause spurious results. In order to ensure a reliable calibration of the magnetometers, the Swarm spacecraft are carrying Absolute Scalar Magnetometers (ASM). These are used as references for validating the vector field data. Despite these precautions, there may be differences in the magnetic field accuracies. Uncertainties in spacecraft positioning and timing are small. In case of Swarm both quantities are based on GPS navigation solutions and thus do not contribute to the error budget. 
Formal uncertainty: For our error estimate we make use of the Swarm specifications. It is required that the difference in magnetic field accuracy between two Swarm spacecraft shall not exceed $1 \mathrm{nT}$ in any component. This value can be inserted in Eq. (6.7), assuming that Swarm A readings are too large by $1 \mathrm{nT}$ with respect to Swarm C. The contributions from the second and fourth term in brackets cancel each other, because the bias can be assumed constant over the $5 \mathrm{~s}$ difference between the first and second pair of readings. Only the first and third term in brackets contribute to the uncertainty. Also here we can set, as a reasonable assumption, $d l_{1}=d l_{3}, d l_{2}$ $=d l_{4}$ and the area $A=d l_{1} . d l_{2}$. With that we obtain a resulting error of radial current density, $\Delta j_{r}$, according to Eq. (6.7):

$$
\Delta j_{r}=\frac{2 n T d l_{1}}{2 \mu_{0} d l_{1} d l_{2}}
$$

Since the zonal separation between the satellite pair varies with the latitude $\beta$, $d l_{2}=155 \mathrm{~km} \cos (\beta)$, the obtained formal uncertainty ranges from $5 \mathrm{nA} / \mathrm{m}^{2}$ at the equator to $75 \mathrm{nA} / \mathrm{m}^{2}$ at $86^{\circ}$ latitude. Beyond $86^{\circ}$ no radial currents are determined. The resulting distribution of the uncertainty with changing latitude is shown in Fig. 6.10 for both radial current and field-aligned current estimates.

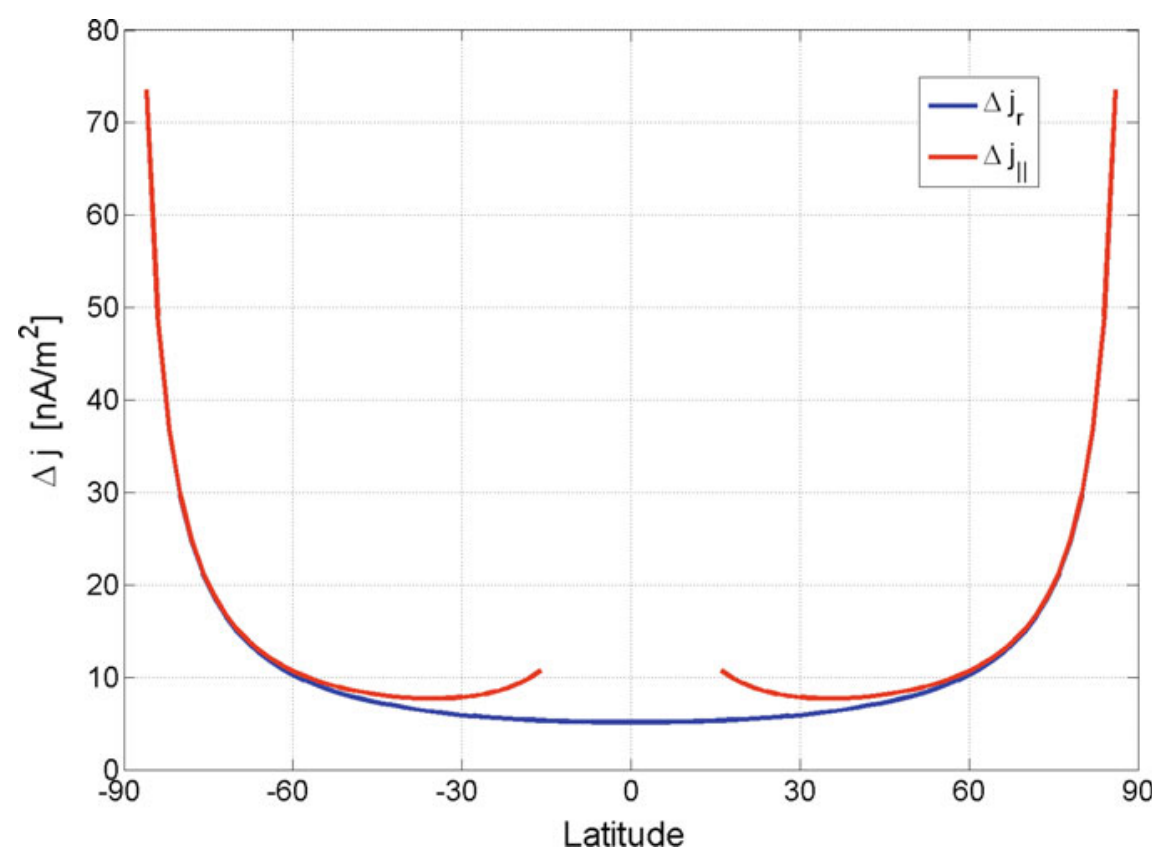

Fig. 6.10 Latitude-dependent variations of the radial current (blue) and FAC (red) estimates. At low latitudes FACs are not calculated due to increasing uncertainties (modified after Ritter et al. 2013) 
Divergence of magnetic field: In other missions, such as Cluster or MMS, the divergence of the magnetic field at the four measurement points has frequently been used as an indicator for the quality of the current estimates. In the case of Swarm, only measurements within a plane are available. Therefore it is not obvious what we can learn from the $2 \mathrm{D}$ divergence derived from the magnetic fields at the four corner points.

We have tested the response of the 2D divergence to several current configurations. Only for the ideal case of measurements within a homogeneous FAC sheet, as shown in Fig. 6.9 (top), the divergence vanishes. Already when part of the measurement quad is located outside the FAC sheet (e.g. Fig. 6.9, bottom) div $B$ attains significant values, and their size is dependent on the orientation angle, $\varphi$. In spite of non-vanishing div $B$ values the dual-SC technique delivers in this case reliable results, as demonstrated above.

We applied also the 2D divergence calculation to actual measurements, but did not attain convincing relations between FAC quality and div $B$ values. In those cases an additional complication arises. Magnetic fields from remote current systems (that can be expressed by scalar potentials) contribute to the $2 \mathrm{D}$ divergence. Conversely, they have little influence on the results of our dual-SC FACs. This difference in characteristics further reduces the significance of $\operatorname{div} B$ for qualifying the FAC results. As a consequence we refrained from using $\operatorname{div} B$ as a quality indicator.

Low latitudes: Field-aligned currents are estimated reliably at auroral latitudes, as shown above. A different situation is found at low latitudes. Here, the integration quad is quite elongated, as shown in Fig. 6.11. Due to the orbital geometry the east-west separation between Swarm A and C, $d l_{2}$, increases to $155 \mathrm{~km}$. This distance is more than 4 times larger than $d l_{l}(38 \mathrm{~km})$. Such a measurement geometry is not consistent with several FAC types existing at low and middle latitudes. An example of FAC estimates is shown in Fig. 6.12. Slightly poleward of $15^{\circ}$ MLat the two spacecraft pass an inter-hemispheric, bipolar FAC structure, with currents directed southward on lower and northward at slightly higher L-shells. In the southern hemisphere this FAC pair is recorded only by Swarm A and 10 min later it is passed by Swarm C in the northern hemisphere. This clearly indicates that the zonal extension of this particular FAC structure is smaller than the satellites separation $(155 \mathrm{~km})$. Consequently, the dual-SC

Fig. 6.11 Sketch of measurement quad. At low latitudes the zonal spacecraft separation $\left(\mathrm{dl}_{2}=155 \mathrm{~km}\right)$ is 4 times larger than the along-track element $(\mathrm{d} 11=$ $38 \mathrm{~km}$ ). This elongated measurement geometry tends to underestimate FAC densities of certain features

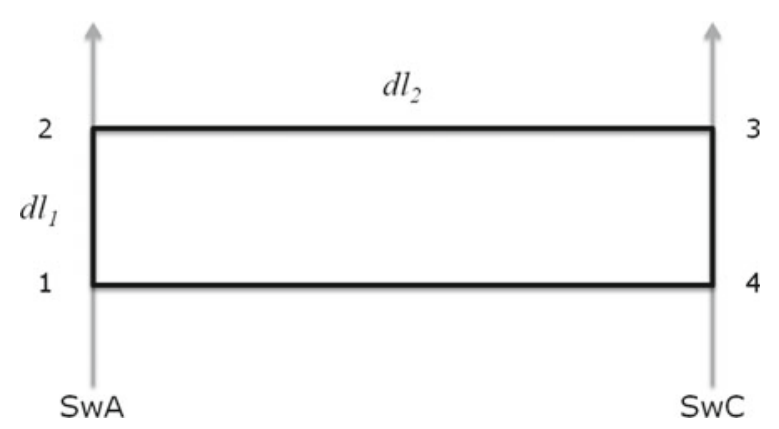



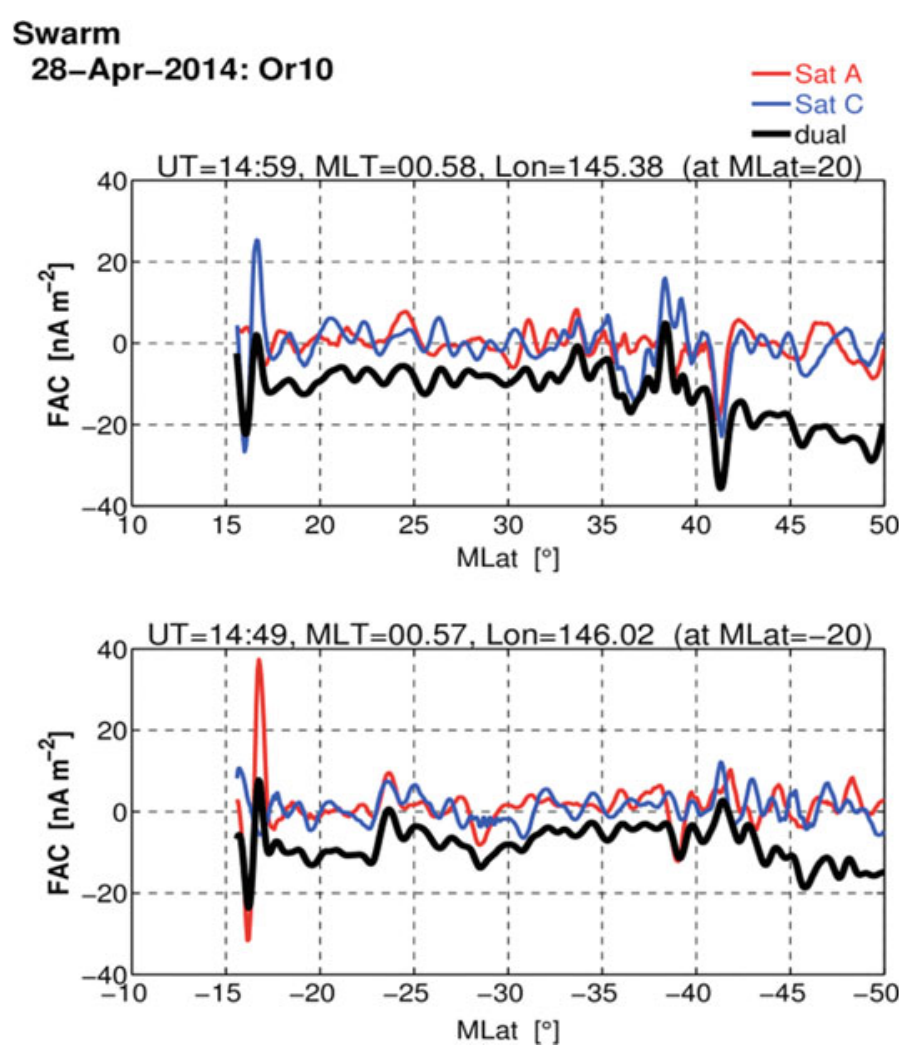

Fig. 6.12 Example of FAC estimates at low and middle latitudes. Near $15^{\circ}$ MLat a conjugate FAC feature is observed in the southern hemisphere only by Swarm A and in the northern only by Swarm C. Consequently, the dual-SC method underestimates the current density. Conversely, large-scale inter-hemispheric FACs are sensed by the dual-SC method at mid-latitudes in both hemispheres but missed by the single satellites

approach returns current densities that are too small. From studies of accompanying observations, Park et al. (2015) have identified this event as medium-scale travelling ionospheric disturbance (MSTID). Numerical model simulations of these phenomena, e.g. by Yokoyama and Hysell (2010) predict elongated inter-hemispheric FAC sheets, approximately $50 \mathrm{~km}$ wide in zonal direction and several hundred $\mathrm{km}$ long, which are tilted by about $30^{\circ}$ away from the meridian. These features propagate at a speed of around $100 \mathrm{~m} / \mathrm{s}$ towards the equator and towards earlier local times. Our low-pass filter does not efficiently remove such elongated FAC sheets. This example shows that, at low latitudes, not all FAC features may be quantified correctly with the Swarm dual-SC approach. In these regions, individual measurements from the satellite pair should be used for checking the dimensions of the features.

At low and middle latitudes there exist also large-scale inter-hemispheric FACs. These can be detected very well with the dual-SC approach. In Fig. 6.12 there is clear evidence for a negative current density at mid-latitude in both hemispheres, which 
is missed by the single-satellite estimates. This indicates an inter-hemispheric FAC flowing from northern to southern hemisphere. The rather low amplitude, about 10 $\mathrm{nA} / \mathrm{m}^{2}$, is quite typical for the night-time sector, but is well resolvable. Lühr et al. (2015b) performed a systematic study of inter-hemispheric large-scale FACs, based on Swarm measurements, revealing a number of characteristic properties of these low-latitude currents.

\subsection{Summary and Conclusions}

The constellation mission Swarm can efficiently be used for determining currents in the ionosphere. In particular the magnetic field measurements from the lower pair, Swarm A \& C flying side-by-side, provide a good basis for a reliable estimate of the radial current component. The underlying method is Ampère's ring integral. Pairs of field readings from both satellites separated along the track by $5 \mathrm{~s}$ are used to define a quad in space. Around this quad the integration is performed to derive the mean value of current density flowing through the encircled area. At auroral latitudes the separation of measurement points is typically $50 \mathrm{~km}$, therefore only large-scale FACs with horizontal scales $>150 \mathrm{~km}$ are considered. Smaller structures are removed by filtering.

Estimates of the formal uncertainties range from $5 \mathrm{nA} / \mathrm{m}^{2}$ at the equator to about $100 \mathrm{nA} / \mathrm{m}^{2}$ near the poles. With this high resolution a whole range of current features can be investigated.

First statistical studies confirm a reliable detection of FAC structures in the auroral oval. Special features like NBZ currents are resolved much clearer than could be achieved previously by single-satellite measurements. In particular in the polar cap region, the dual-SC approach provides a much more detailed picture of FAC distribution.

The high sensitivity of the dual-SC approach also allows for systematic investigations of week inter-hemispheric FACs at middle latitudes. These are closely related to the solar quiet, $\mathrm{Sq}$, current systems and show clear seasonal and longitudinal variations.

The Swarm pair is even able to resolve the wind-driven radial currents over the magnetic equator (see Lühr et al. 2016b). Their intensity and change in direction between noon and evening has clearly been recovered by means of the dual-SC approach.

The presented examples show that the routinely generated radial currents (FACs) densities enable a number of interesting studies. An important constrains to be considered is the size of the current structure. Horizontal scales of the FAC structures interpreted have to be at least twice as large as the gross dimensions of the measurement quad. This requirement can be checked by comparing the magnetic field measurements at satellites Swarm A and C.

All phenomena mentioned here call for further, more detailed investigations. For that purpose more data are necessary. The Swarm constellation needs approximately 
5 years for covering all local times evenly during all seasons. The Swarm mission thus offers the opportunity for a number of interesting systematic FAC studies in future when the present Swarm A \& C configuration is maintained at least until May 2019.

Acknowledgements The authors are grateful to Jaeheung Park and Ingo Michaelis for fruitful scientific discussions and continuous support during the development of the processing code. The European Space Agency (ESA) is acknowledged for providing the Swarm data and for financially supporting the work on developing the Swarm Level 2 product "FAC". The authors thank the International Space Science Institute in Bern, Switzerland, for supporting the ISSI Working Group: "Multi-Satellite Analysis Tools Ionosphere", in which frame this study was performed. The Editors thanks Guan Le for her assistance in evaluating this chapter.

\section{References}

Dunlop, M.W., D.J. Southwood, K.-H. Glassmeier, and F.M. Neubauer. 1988. Analysis of multipoint magnetometer data. Advances in Space Research 8: 273-277.

Dunlop, M.W., A. Balogh, K.-H. Glassmeier, and P. Robert. 2002. Four-point Cluster application of magnetic field analysis tools: The Curlometer. Journal Geophysical Research 107 (A11): 1384. https://doi.org/10.1029/2001JA005088.

Dunlop, M.W., and A. Balogh. 2005. Magnetopause current as seen by Cluster. Annales Geophysicae 23: 901-907.

Dunlop, M.W., Y.-Y. Yang, J.-Y. Yang, H. Lühr, C. Shen, N. Olsen, P. Ritter, Q.-H. Zhang, J.-B. Cao, H.-S. Fu, and R. Haagmans. 2015. Multispacecraft current estimates at Swarm. Journal of Geophysical Research: Space Physics 120: 8307-8316. https://doi.org/10.1002/2015JA021707.

Dunlop, M.W., S. Haaland, P.C. Escoubet, and X.-C. Dong. 2016. Commentary on accessing 3-D currents in space: Experiences from cluster. Journal of Geophysical Research: Space Physics 121: 7881-7886. https://doi.org/10.1002/2016JA022668.

Eastwood, J.P., et al. 2016. Ion-scale secondary flux ropes generated by magnetopause reconnection as resolved by MMS. Geophysical Reseach Letters 43: 4716-4724. https://doi.org/10.1002/ 2016 GL068747.

Finlay, C.C., N. Olsen, S. Kotsiaros, N. Gillet, and L. Tøffner-Clausen. 2016. Recent geomagnetic secular variation from Swarm and ground observatories as estimated in the CHAOS-6 geomagnetic field model. Earth Planets Space 68: 112. https://doi.org/10.1186/s40623-016-0486-1.

Gjerloev, J.W., S. Ohtani, T. Iijima, B. Anderson, J. Slavin, and G. Le. 2011. Characteristics of the terrestrial field-aligned current system. Annales Geophysicae 29: 1713-1729.

Le, G., Y. Wang, J.A. Slavin, and R.J. Strangeway. 2009. Space Technology 5 multipoint observations of temporal and spatial variability of field-aligned currents. Journal Geophysical Research 114: A08206. https://doi.org/10.1029/2009JA014081.

Lühr, H., J. Warnecke, and M.K.A. Rother. 1996. An algorithm for estimating field-aligned currents from single spacecraft magnetic field measurements: A diagnostic tool applied to Freja satellite data. IEEE Transactions on Geoscience and Remote Sensing 34: 1369-1376.

Lühr, H., J. Park, J.W. Gjerloev, J. Rauberg, I. Michaelis, J.M.G. Merayo, and P. Brauer. 2015a. Fieldaligned currents' scale analysis performed with the swarm constellation. Geophysical Reseach Letters 42: 1-8. https://doi.org/10.1002/2014GL062453.

Lühr, H., G. Kervalishvili, I. Michaelis, J. Rauberg, P. Ritter, J. Park, J.M.G. Merayo, and P. Brauer. 2015b. The inter-hemispheric and F-region dynamo currents revisited with the Swarm constellation. Geophysical Reseach Letters 42: 3069-3075. https://doi.org/10.1002/2015GL063662.

Lühr, H., T. Huang, S. Wing, G. Kervalishvili, J. Rauberg, and H. Korth. 2016. Filamentary fieldaligned currents at polar cap region during northward interplanetary magnetic field derived with the Swarm constellation. Annales Geophysicae 34: 901-915. https://doi.org/10.5194/angeo-34901-2016. 
Park, J., H. Lühr, G. Kervalishvili, J. Rauberg, I. Michaelis, C. Stolle, and Y.-S. Kwak. 2015. Nighttime magnetic field fluctuations in the topside ionosphere at midlatitudes and their relation to medium-scale traveling ionospheric disturbances: The spatial structure and scale sizes. Journal of Geophysical Research: Space Physics. 120. https://doi.org/10.1002/2015ja021315.

Ritter, P., and H. Lühr. 2006. Curl-B technique applied to Swarm constellation for determining field-aligned currents. Earth Planets Space 58: 463-476.

Ritter, P., H. Lühr, and J. Rauberg. 2013. Determining field-aligned currents with the Swarm constellation mission. Earth Planets Space 65: 1285-1294. https://doi.org/10.5047/eps.2013.09.006.

Slavin, J.A., G. Le, R.J. Strangeway, Y. Wang, S.A. Boardsen, M.B. Moldwin, and H.E. Spence. 2008. Space Technology 5 multi-point measurements of near-Earth magnetic fields: Initial results. Geophysical Reseach Letters 35: L02107. https://doi.org/10.1029/2007GL031728.

Sonnerup, B.U.O., and L.J. Cahill. 1967. Magnetopause structure and attitude from explorer 12 observations. Journal Geophysical Research 72: 171-183.

Vallat, C., I. Dandouras, M. Dunlop, A. Balogh, E. Lucek, G.K. Parks, M. Wilber, E.C. Roelof, G. Chanteur, and H. Rème. 2005. First current density measurements in the ring current region using simultaneous multispacecraft CLUSTER-FGM data. Annales Geophysicae 23: 1849-1865.

Vennerstrøm S., E. Friis-Christensen, H. Lühr, T. Moretto, N. Olsen, C. Manoj, P. Ritter, L. Rastätter, A. Kuvshinov, and S. Maus. 2005. The impact of combined magnetic and electric field analysis and of ocean circulation effects on Swarm mission performance, ESA contract No 3-10901/03/NL/CB, DSRI Report 2/2004.

Vogt, J., E. Sorbalo, M. He, and A. Blagau. 2013. Gradient estimation using configurations of two or three spacecraft. Annales Geophysicae 31: 1913-1927. https://doi.org/10.5194/angeo-31-19131927-2013.

Wang, Y., G. Le, J.A. Slavin, S.A. Boardsen, and R.J. Strangeway. 2009. Space Technology 5 measurements of auroral field-aligned current sheet motion. Geophysical Reseach Letters 36: L02105. https://doi.org/10.1029/2008GL035986.

Yokoyama, T., and D.L. Hysell. 2010. A new midlatitude ionosphere electrodynamics coupling model (MIECO): Latitudinal dependence and propagation of medium-scale traveling ionospheric disturbances. Geophysical Reseach Letters 37: L08105. https://doi.org/10.1029/2010GL042598.

Zhao, C., C.T. Russell, R.J. Strangeway, S.M. Petrinec, W.R. Paterson, M. Zhou, B.J. Anderson, W. Baumjohann, K.R. Bromund, M. Chutter, D. Fischer, G. Le, R. Nakamura, F. Plaschke, J.A. Slavin, R.B. Torbert, and H.Y. Wei. 2016. Force balance at the magnetopause determined with MMS: Application to flux transfer events. Geophysical Reseach Letters 43: 11941-11947. https:// doi.org/10.1002/2016GL071568.

Open Access This chapter is licensed under the terms of the Creative Commons Attribution 4.0 International License (http://creativecommons.org/licenses/by/4.0/), which permits use, sharing, adaptation, distribution and reproduction in any medium or format, as long as you give appropriate credit to the original author(s) and the source, provide a link to the Creative Commons license and indicate if changes were made.

The images or other third party material in this chapter are included in the chapter's Creative Commons license, unless indicated otherwise in a credit line to the material. If material is not included in the chapter's Creative Commons license and your intended use is not permitted by statutory regulation or exceeds the permitted use, you will need to obtain permission directly from the copyright holder.

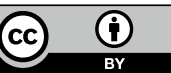

